\title{
The many faces of hydatid disease
}

A Lawson, MB BCh
Department of Radiology, Groote Schuur Hospital, Cape Town
A Parrish, MB ChB, DA(SA), FCP(SA),
MMed(Med), MMedSci(ClinEpi)
Department of Medicine, Cecilia Makiwane Hospital, East London

\section{Case report}

A 35-year-old man presented with a right chest wall mass. Chest radiographs revealed pleural thickening underlying a soft tissue mass which obscured the 5th to 7 th ribs, and an expansile osteolytic lesion of the 7th rib. Ultrasound examination demonstrated a fluid-filled cystic lesion of the chest wall with intrathoracic extension.

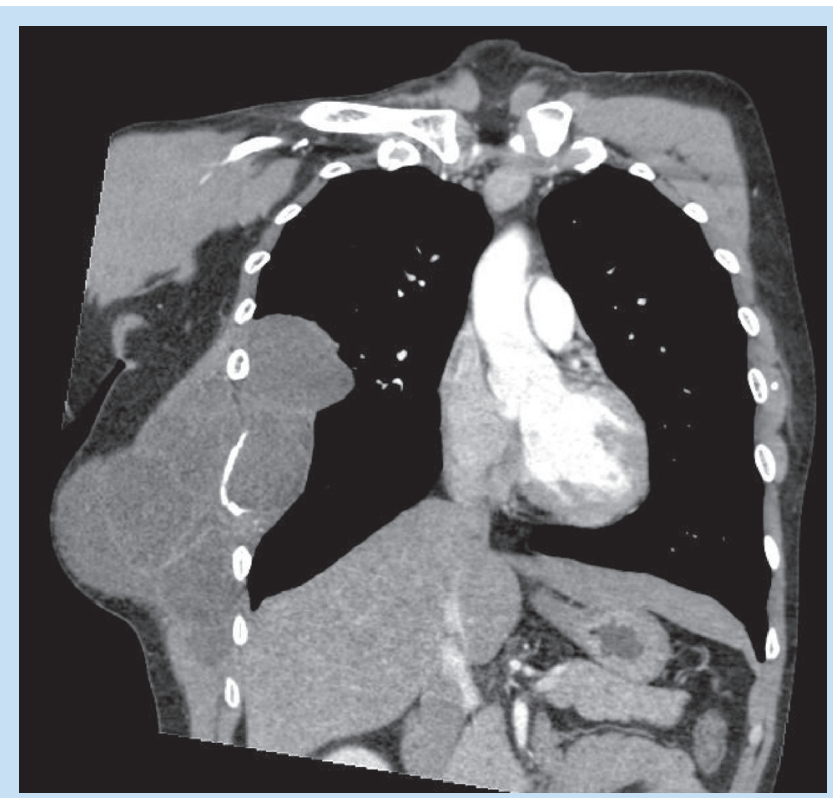

Fig. 1. CT coronal reconstruction.

A computed tomographic (CT) scan of the chest and upper abdomen revealed a fluid-filled cystic structure protruding from the chest wall into the subcutaneous tissues. An abutting cyst extended within the thoracic cavity but did not involve the lung parenchyma. Three-dimensional reconstruction of the bony elements revealed an expanding bony lesion with multiple cysts in the 7th rib, surrounded by the large fluidfilled cyst. No hepatic cysts were demonstrated.

An ELISA (enzyme linked immuno-sorbent assay) test for Echinococcus granulosis was positive to a titre of 1:256.

The patient is currently on a course of albendazole chemotherapy and is followed up at the surgical outpatient clinic.

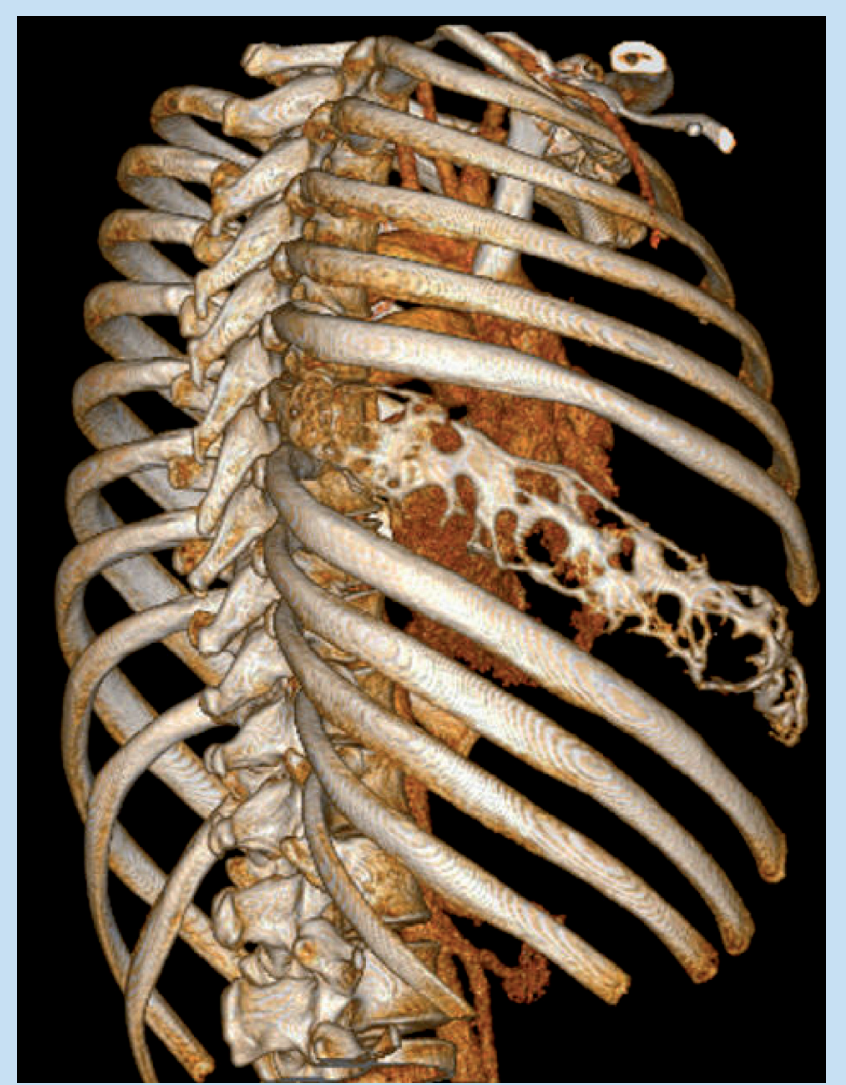

Fig. 2. CT 3-D reconstruction.

\section{Discussion}

A hydatid cyst may be found in almost any part of the body; however, a primary chest wall cyst is rare. ${ }^{1}$ This patient had an extra-pulmonary but intrathoracic hydatid cyst that presented as a chest wall tumour.

A possible mechanism of primary hydatid disesase of the chest wall may be for the embryo to pass through the duodenal wall into either the portal vein or the periduodenal and perigastric lymphatics, which connect to the thoraco-mediastinal lymphatics and the thoracic duct. ${ }^{2}$ This mechanism may explain the development of primary chest wall hydatid disease in the absence of pulmonary or hepatic cysts.

The natural course of costal echinococcosis starts when the larvae lodge in the rib and buds start vegetating out of the mother cyst to produce a multilocular cavity. This process invades the spongiosa of the bone. The primary rib lesion is multiloculated and osteolytic and continues to grow slowly. The lesion may then involve adjacent organs such as vertebrae, pleura and soft tissues. ${ }^{3}$ If this lesion breaks through the cortical portion of the rib, it produces a soft tissue mass. ${ }^{4}$ The posterior ends of the ribs are most commonly involved in costal echinococcosis. Cysts grow along the long axis of the rib, causing expansion of the cortex 
where they meet more resistance from the solid cortical portion of the rib.

The differential diagnosis of such a radiographic picture includes giant cell tumour, osteolytic metastases, plasmacytoma, aneurysmal bone cyst and cystic neurofibromas.

Biopsy is generally considered to be contraindicated in echinococcosis owing to fear of dissemination of scolices and other potentially fatal acute anaphylactic reactions. However, aspiration cytology has been suggested as being safe and the procedure of choice in suspected cases of skeletal echinococcosis.

\section{Conclusion}

A case of primary hydatid disease arising in a rib is reported. This potentially curable condition should be considered in the differential diagnosis of both abdominal wall masses and mass lesions identified on chest radiographs.

1. Findikcioglu A, Kilic D, Canpolat T, et al. Primary hydatid disease of the chest wall. Ann Thorac Cardiovas Surg 2007; 13(3): 203-205.

2. Heath DD. The migration of oncospheres of Taenia pisiformis, T. serialis and Echinococcus granulosus within the intermediate host. Int J Parasitol 1971; 1: 145-152.

3. Beggs I. The radiology of hydatid disease. Am J Roentgenol 1985; 145: 639-648.

4. Bonakdarpour A, Ali Zadeh YF, Maghssoudi H, et al. Costal echinococcosis. Report of six cases and review of literature. Am J Roentgenol 1973; 118: 371-377.

\section{The role of the expert witness}

\section{(continued from page 2)}

'In sum, we pathologists [health care workers] have an obligation to ourselves and to our specialty to set standards for behaviour in the courtroom that are consistent with our behavior in the rest of our professional lives. That behavior should be elevated and elevating. The behavior of pathologists in the courtroom should be no different from their behavior in the laboratory - but very different from the behavior of all too many lawyers in the courtroom.'

Hugh Johnson puts it even more strongly: 'The fundamental difference between the doctor and the lawyer is in their approach to the case. A lawyer is not interested in the real truth in its entirety but only such part as pertains to his client's case. The lawyer obviously must take sides. The expert pathologist must not.'

These are harsh words, but the expert witness should always be reminded of the prescribed oath that is taken: 'I swear that the evidence I am about to give is the truth, the whole truth and nothing but the truth. So help me God'. Withholding important facts, especially quoting selectively from the literature with deliberate omission and withholding of important facts from the court, is as good as outright lying, and tantamount to perjury.

\section{Conclusion}

To conclude with some wise words from Bernard Ackerman: ${ }^{6}$

'How should a conscientious pathologist [any health care worker] deal intellectually and emotionally with an error he has made? First and foremost, he must acknowledge it promptly and unequivocally. Second, he must attempt to determine why the error was made, and strive to learn from it. ... How can we as ... [health care workers]... put this distasteful, disagreeable and often demeaning but very real aspect of our (professional) lives in perspective?
'Perhaps it can be done if each of us acknowledges to himself and tacitly to others that, although we are capable professionals, we nonetheless have human limitations, inadequacies and failings. We are bound to make mistakes, yet we must try to perform as best as we can within the confines of our human limitations. ${ }^{6}$

In her letter to the SAMJ, van der Heyde, a young forensic pathologist from Cape Town, who has experienced difficult interrogation during inquests, highlights the difficulty in persuading medical doctors to assist the prosecutor and give expert evidence at inquests and trials: 'The Medical Protection Society (MPS) legal team .... has access to specialists in different fields of medicine who are paid well to give expert testimony and assist the MPS in defending their clients ... .Court proceedings are generally unpleasant for health care workers. However, does the medical profession not have an ethical responsibility ... [to give unbiased evidence for justice and the truth]..., given that the standard by which conduct is tested at an inquest is based on what the reasonable doctor would do?"7

Emeritus Professor G J (Deon) Knobel

Formerly of the Department of Forensic Medicine

University of Cape Town

1. Knobel GJ. Varied facets of forensic medicine. Inaugural lecture 13 August 1986: New series no. 125.

2. Johnson H. The pathologist as expert witness. Med Leg J 1986; 54, part 1: 26 - 41.

3. Gillon R. Doctors and patients. BMJ 1986; 292: 466-469.

4. Lundberg GD. Expert witness for whom? JAMA 1984: 252: 251.

5. Ackerman B. The pathologist in the courtroom: Peer review is needed. Hum Pathol 1985; 16: 761-762.

6. Ackerman A Bernard. Accountability of pathologists. Am I Derm Path 1985; 7; 305-306.

7. van der Heyde Y. Medical responsibility at inquests. S Afr Med J 2008; 98: 820-822. 